\title{
Primer containing dimethylaminododecyl methacrylate kills bacteria impregnated in human dentin blocks
}

\author{
Chen Chen ${ }^{1,2}$, Lei Cheng ${ }^{1}$, Michael D Weir ${ }^{2}$, Nancy J Lin ${ }^{3}$, Sheng Lin-Gibson ${ }^{3}$, Xue-Dong Zhou ${ }^{1}$ \\ and Hockin $\mathrm{HK} \mathrm{Xu}^{2,4,5}$
}

Antibacterial dimethylaminododecyl methacrylate (DMADDM) was recently synthesized. The objectives of this study were to: (1) investigate antibacterial activity of DMADDM-containing primer on Streptococcus mutans impregnated into dentin blocks for the first time, and (2) compare the antibacterial efficacy of DMADDM with a previous quaternary ammonium dimethacrylate (QADM). Scotchbond Multi-Purpose (SBMP) bonding agent was used. DMADDM and QADM were mixed into SBMP primer. Six primers were tested: SBMP control primer P, P+2.5\% DMADDM, P+5\% DMADDM, P+7.5\% DMADDM, P+10\% DMADDM, and P+10\% QADM. S. mutans were impregnated into human dentin blocks, and each primer was applied to dentin to test its ability to kill bacteria in dentinal tubules. Bacteria in dentin were collected via a sonication method, and the colony-forming units (CFU) and inhibition zones were measured. The bacterial inhibition zone of $P+10 \%$ DMADDM was 10 times that of control primer $(P<0.05)$. CFU in dentin with $\mathrm{P}+10 \%$ DMADDM was reduced by three orders of magnitude, compared with control. DMADDM had a much stronger antibacterial effect than QADM, and antibacterial efficacy increased with increasing DMADDM concentration. Dentin shear bond strengths were similar among all groups $(P>0.1)$. In conclusion, antibacterial DMADDM-containing primer was validated to kill bacteria inside dentin blocks, possessing a much stronger antibacterial potency than the previous QADM. DMADDM-containing bonding agent was effective in eradicating bacteria in dentin, and its efficacy was directly proportional to DMADDM mass fraction. Therefore, DMADDM may be promising for use in bonding agents as well as in other restorative and preventive materials to inhibit bacteria.

International Journal of Oral Science (2016) 8, 239-245; doi:10.1038/ijos.2016.43; published online 4 November 2016

Keywords: antibacterial bonding agent; dental restoration; dentin bond strength; dimethylaminododecyl methacrylate; killing bacteria in dentin; Streptococcus mutans

\section{INTRODUCTION}

Dental caries is the destruction of dental hard tissues caused by acidic products from bacterial fermentation of dietary carbohydrates. ${ }^{1-2}$ Approximately 200 million tooth cavity restorations were performed annually in the United States, costing about $\$ 40$ billion per year. ${ }^{3}$ Resin composites are popular tooth cavity filling materials due to their esthetics and direct-filling capabilities. The chemical, physical, and mechanical properties of composites have been improved significantly. ${ }^{4-11}$ Nonetheless, about half of all restorations fail in less than 10 years, with secondary caries as one of the primary reasons. ${ }^{12-15}$ Therefore, it would be highly desirable to improve the materials to reduce secondary caries and restoration failure. ${ }^{11-15}$

The concept of completely removing carious dentin during cavity preparation is no longer recommended. ${ }^{13-14}$ Residual bacteria remaining in dentinal tubules of the relatively healthy part of the dentin make it impossible to achieve caries-free cavities through the complete caries removal approach. ${ }^{13,16}$ Furthermore, minimally invasive techniques propose the least invasive surgical approaches to remove carious lesion, in order to preserve more tooth structure and protect the pulpal vitality. ${ }^{14,17-18}$ Several methods were developed to precisely remove caries-infected tissue while achieving maximum preservation of caries-affected tissues. ${ }^{14}$ However, while preserving more tooth structure is meritorious, it will also leave more residual bacteria in the prepared cavity. ${ }^{19}$ Therefore, it would be beneficial to develop antibacterial primers that can be applied into the tooth cavity to eradicate or significantly reduce bacterial load in the dentin, thereby minimizing the adverse effects of residual bacteria.

Adhesives are used to bond composite restorations to tooth structures. Previous studies have improved the adhesive compositions, bond strength, and the durability of the bonded interface. ${ }^{20-26}$

${ }^{1}$ State Key Laboratory of Oral Diseases, West China School of Stomatology, Sichuan University, Chengdu, China; ${ }^{2}$ Biomaterials \& Tissue Engineering Division, Department of Endodontics, Periodontics and Prosthodontics, University of Maryland Dental School, Baltimore, USA; ${ }^{3}$ Biomaterials Group, Biosystems and Biomaterials Division, National Institute of Standards \& Technology, Gaithersburg, USA; ${ }^{4}$ Center for Stem Cell Biology \& Regenerative Medicine, University of Maryland School of Medicine, Baltimore, USA and ${ }^{5}$ Department of Mechanical Engineering, University of Maryland, Baltimore County, USA

Correspondence: Professor XD Zhou, State Key Laboratory of Oral Diseases, West China College of Stomatology, Sichuan University, No. 14, Section 3, Renmin South Road, Chengdu 610041, China

E-mail: zhouxd@scu.edu.cn

Professor HHK Xu, Biomaterials \& Tissue Engineering Division, Department of Endodontics, Periodontics and Prosthodontics, University of Maryland School of Dentistry, Baltimore MD 21201, USA

E-mail: hxu@umaryland.edu

Accepted 9 December 2015 
In order to impart an antibacterial property to inhibit biofilm growth, quaternary ammonium methacrylates (QAMs) were synthesized and incorporated into dental resins. ${ }^{27-31}$ Novel bonding agents containing 12-methacryloyloxydodecyl-pyridinium bromide (MDPB) were developed and showed a strong antibacterial activity. ${ }^{27,32}$ Other investigators synthesized methacryloxyl ethyl cetyl dimethyl ammonium chloride (DMAE-CB) for incorporation into resins. ${ }^{33}$ Besides bonding agents, dental composites containing nanoparticles of quaternary ammonium polyethylenimine were also developed with antibacterial functions. ${ }^{34}$ Recently, a quaternary ammonium dimethacrylate (QADM) was synthesized and incorporated into resins, showing effective inhibition of dental plaque microcosm biofilm growth. ${ }^{35}$

With the purpose of killing residual bacteria inside dentinal tubules in tooth cavities, a previous study showed that a primer containing MDPB killed bacteria that had been impregnated into a dentin block. $^{16}$ Recently, another primer containing QADM was also demonstrated to kill Streptococcus mutans impregnated into human dentin blocks. ${ }^{36}$ Therefore, such antibacterial bonding agents are promising to eradicate bacteria in tooth cavities in order to inhibit caries and protect the pulp, and they could be especially beneficial when using minimally invasive procedures. More recently, a new quaternary ammonium monomer dimethylaminododecyl methacrylate (DMADDM) was synthesized and shown to possess a highly potent antibacterial activity. ${ }^{37}$ However, the effect of a DMADDMcontaining primer on the killing of bacteria impregnated into dentin blocks has not been reported.

The objectives of this study were to investigate the effects of a DMADDM-containing primer on the killing efficacy of S. mutans impregnated into dentin in comparison with the previous QADM. The following hypotheses were tested: (1) both DMADDM-containing primer and QADM-containing primer would be effective in killing S. mutan inside dentin, but DMADDM-containing primer would have a higher efficacy than QADM-containing primer in killing bacteria inside dentin; (2) increasing the DMADDM mass fraction in primer would increase the efficacy of killing bacteria inside dentin; (3) the killing of bacteria inside a tooth cavity could be achieved without compromising the dentin shear bond strength, compared with control primer without DMADDM.

\section{MATERIALS AND METHODS}

\section{Synthesis of DMADDM}

DMADDM was synthesized using a modified Menschutkin reaction method, in which a tertiary amine group was reacted with an organo-halide. ${ }^{29,35,37}$ Commercial 1-(dimethylamino)docecane (DMAD; Tokyo Chemical Industry, Tokyo, Japan) and 2-bromoethyl methacrylate (BEMA; Monomer-Polymer and Dajac Labs, Trevose, PA, USA) were used as the tertiary amine and the organo-halide, respectively. First, $10 \mathrm{mmol}$ of DMAD and $10 \mathrm{mmol}$ of BEMA were mixed in a $20 \mathrm{~mL}$ scintillation vial with a magnetic stir bar. The reaction mixture was stirred at $70{ }^{\circ} \mathrm{C}$ for $24 \mathrm{~h} .{ }^{37}$ After the reaction was completed, the ethanol solvent was removed via evaporation. This process yielded DMADDM as a clear, colorless, and viscous liquid. Fourier transform infrared spectroscopy confirmed the reaction and the products in a previous study. ${ }^{37}$

\section{DMADDM incorporation into primer}

Scotchbond Multi-Purpose (3M, St. Paul, MN, USA) was used as the parent bonding system (referred to as SBMP). According to the manufacturer, SBMP etchant contained 37\% (all by mass) phosphoric acid. SBMP primer contained $35 \%-45 \%$ of 2-hydroxyethylmethacrylate (HEMA), $10 \%-20 \%$ of a copolymer of acrylic and itaconic acids, and $40 \%-50 \%$ of water. SBMP adhesive contained $60 \%-70 \%$ of bisphenol A diglycidyl methacrylate (BisGMA), 30\%-40\% of HEMA, tertiary amines and photo-initiator. The present study tested the killing of bacteria via primer, hence DMADDM was incorporated into the primer. The SBMP etchant and adhesive were unmodified. The following experimental bonding systems, which contained SBMP primer mixed with different mass fractions of DMADDM, were tested and compared with the control group:

(1) SBMP primer (P; control).

(2) $\mathrm{P}+2.5 \%$ (by mass) DMADDM.

(3) $\mathrm{P}+5 \%$ DMADDM.

(4) $\mathrm{P}+7.5 \%$ DMADDM.

(5) $\mathrm{P}+10 \%$ DMADDM.

(6) $\mathrm{P}+10 \%$ QADM.

QADM had been developed and investigated in previous studies ${ }^{29,36}$ and served as a comparative control here, to determine the relative antibacterial potency of DMADDM, compared with the previous QADM. These mass fractions were selected following a previous study. ${ }^{38}$

\section{Dentin shear bond strength testing}

The use of extracted human teeth was approved by the University of Maryland Institutional Review Board. Caries-free molars were cleaned and stored in $0.01 \%$ thymol solution. Flat mid-coronal dentin surfaces were prepared by cutting off the tips of crowns with a diamond saw (Isomet, Buehler, Lake Bluff, IL, USA). ${ }^{35,39}$ Each tooth was embedded in a poly-carbonate holder (Bosworth, Skokie, IL, USA). The occlusal surface was ground on 320 grit silicon carbide paper until the occlusal enamel was removed. The dentin surface was etched with the SBMP echant for $15 \mathrm{~s}$ and then washed with water. A primer was applied and rubbed in for $15 \mathrm{~s}$ with a brush-tipped applicator. The solvent was removed with a stream of air for $5 \mathrm{~s}$. Then the adhesive was applied and light-cured for $10 \mathrm{~s}$ (Optilux VCL 401; Demetron Kerr, Danbury, CT, USA). A $1.5 \mathrm{~mm}$-thick stainless-steel iris with a central opening of $4 \mathrm{~mm}$ in diameter was placed on the adhesive-treated dentin surface. $^{40}$ The central opening was filled with a composite (TPH; Caulk/Dentsply, Milford, DE, USA) and light-cured for $60 \mathrm{~s}^{36-37}$ The bonded specimens were stored in water at $37^{\circ} \mathrm{C}$ for 1 day and then used for testing. A chisel connected to a Universal Testing Machine (MTS, Eden Prairie, MN, USA) was aligned to be parallel to the composite-dentin interface. Then a load increasing at a constant rate of $0.5 \mathrm{~mm} \cdot \mathrm{min}^{-1}$ was applied until the dentin surface and composite were separated. Dentin shear bond strength $S$ was calculated as

$$
S=4 P /\left(\pi d^{2}\right)
$$

where $P$ is the load for fracturing the bond, and $d$ is the diameter of the composite. ${ }^{36-37,40}$

\section{Agar disk diffusion test}

S. mutans is a cariogenic bacterium and is the primary causative agent of caries. The use of S. mutans (ATCC 700610; American Type Culture, Manassas, VA, USA) was approved by the University of Maryland Institutional Review Board. Agar disk diffusion test (ADT) was used to examine the antibacterial effect of uncured primers. Samples were prepared by adding $15 \mu \mathrm{L}$ of stock bacteria to $15 \mathrm{~mL}$ of growth medium consisting of brain heart infusion (BHI) broth (Becton Dickenson, Franklin Lakes, NJ, USA) supplemented with $0.2 \%$ sucrose. After $24 \mathrm{~h}$ incubation at $37^{\circ} \mathrm{C}$ with $5 \%$ (by volume) $\mathrm{CO}_{2}, 0.4 \mathrm{~mL}$ of bacteria suspension was swabbed across a BHI agar 
plate ( $4 \mathrm{~mm}$ height and $90 \mathrm{~mm}$ diameter). A primer-impregnated paper disk was prepared by dropping $20 \mu \mathrm{L}$ of a primer into a sterile paper disk with $1.5 \mathrm{~mm}$ thickness and $6 \mathrm{~mm}$ diameter, following previous studies. ${ }^{16,37}$ The primer-impregnated paper disks were placed on agar plates with bacteria and incubated in $5 \% \mathrm{CO}_{2}$ at $37^{\circ} \mathrm{C}$ for $48 \mathrm{~h}$. Bacteria inhibition zone size $=$ (outer diameter of inhibition zone - paper disk diameter)/2, following previous studies. ${ }^{16,37}$

\section{Bacteria impregnation into dentin blocks and scanning electron microscopy examination}

Dentin blocks of $\sim 4 \mathrm{~mm} \times 4 \mathrm{~mm} \times 0.5 \mathrm{~mm}$ were cut from the crown of extracted molars. Dentin blocks were cut at a distance of $\sim 1 \mathrm{~mm}$ away from the pulp, with one surface facing the pulp, and the other surface facing the occlusal enamel. The dentin block was ground on a 1500 grit silicon carbide abrasive paper to reduce the thickness to $\sim 200 \mu \mathrm{m} .{ }^{16,36}$ The dentin surface was treated with $37 \%$ phosphoric acid for $3 \mathrm{~min}$ and then thoroughly rinsed with distilled water for $60 \mathrm{~s}$ to remove the smear layer. ${ }^{41}$ The dentin samples were then sterilized with ethylene oxide (Anprolene AN 74i; Andersen, Haw River, NC, USA).

To simulate bacterial colonization in carious dentin, a $2 \mu \mathrm{L}$ aliquot of $S$. mutans suspension, having a concentration of $10^{10} \mathrm{CFU}$ per $\mathrm{mL}$ in BHI, was placed on the surface of the dentin block for $10 \mathrm{~min}$ for infiltration, following previous studies. ${ }^{16,36}$ To prepare specimens for scanning electron microscopy (SEM) examination, first, the dentin blocks were immersed in $1 \%$ glutaraldehydein phosphate-buffered saline (PBS) at $4{ }^{\circ} \mathrm{C}$ for $4 \mathrm{~h}$. Then, graded ethanol dehydrations were applied to the dentin. Finally, the dentin blocks were rinsed with $100 \%$ hexamethyldisilazane twice, and sputter-coated with gold (Quanta 200; FEI, Hillsboro, OR, USA). Dentin blocks with and without bacteria impregnation were both examined. ${ }^{36}$

\section{Bacteria viability staining assay}

Two microliters of $S$. mutans suspension at $10^{10} \mathrm{CFU}$ per $\mathrm{mL}$ was placed on a dentin block to infiltrate into dentin, as described above. Then a primer was applied to the dentin and left for $20 \mathrm{~s}$. Two microliters of primer was used for each dentin block. Six dentin blocks were used to test each primer $(n=6)$. The dentin blocks were then stained using a live/dead bacterial viability kit (Molecular Probes, Eugene, OR, USA) following the manufacturer's protocol. Live bacteria were stained with Syto 9 to show a green fluorescence. Bacteria with compromised membranes were stained with propidium iodide to show a red fluorescence. The stained samples were imaged via an epifluorescence microscope (Eclipse TE2000-S; Nikon, Melville, NY, USA)..$^{37,42}$

\section{Method to harvest bacteria from inside dentin blocks}

A $2 \mu \mathrm{L}$ S. mutans suspension at $10^{10} \mathrm{CFU}$ per $\mathrm{mL}$ in BHI was impregnated into a dentin block as described above. ${ }^{16,36}$ Each impregated dentin block was placed into a tube with $2 \mathrm{~mL}$ of cysteine peptone water $(\mathrm{CPW})$. Then the bacteria were harvested by sonication $(3510 \mathrm{R}-$ MTH; Branson Ultrasonics, Danbury, CT, USA) at a frequency of $40 \mathrm{kHz}$ for $5 \mathrm{~min}$ and vortexing at the maximum speed for $20 \mathrm{~s}$ using a vortex mixer (Fisher, Pittsburgh, PA, USA), following a previous study. ${ }^{36}$ The harvested bacteria were serially diluted in CPW and plated on BHI agar plates. ${ }^{36}$ The agar plates were incubated at $37^{\circ} \mathrm{C}$ with $5 \%$ $\mathrm{CO}_{2}$ for 3 days, and the number of colonies was counted on the plates. This yielded the CFU counts harvested from the dentin block by the sonication method. ${ }^{36}$

To examine whether the sonication method harvested all the bacteria inside the dentin, the dentin after sonication was then cut into small pieces and homogenized in $500 \mu \mathrm{L}$ of CPW, following previous studies. ${ }^{16,36}$ The cutting and homogenizing method was previously shown to harvest the bacteria in dentin blocks. ${ }^{16,36}$ The solution with small dentin pieces was vortexed using the vortex mixer at maximum speed for $20 \mathrm{~s} .{ }^{16,36}$ The purpose was to harvest any residual bacteria that remained in the dentin after the sonication method. The homogenized solution was plated on agar plates and incubated for 3 days, and the colonies were counted. If the residual $\mathrm{CFU}$ was negligible compared with the CFU from the sonication method, it would indicate that the sonication method could effectively harvest the bacteria from the blocks. The rationale for this experiment was that the sonication method for harvesting the bacteria in dentin is a simpler method. If the sonication method could effectively harvest bacteria from the dentin, then the steps of cutting and homogenizing the dentin block could be avoided.

Effects of primers on the killing of $S$. mutans impregnated in dentin As described above, $2 \mu \mathrm{L}$ of $S$. mutans at $10^{10} \mathrm{CFU}$ per $\mathrm{mL}$ was placed on a dentin block to impregnate the bacteria into dentinal tubules. Then a primer was applied to the dentin. The dentins were used for CFU measurement using the sonication method. ${ }^{36}$ Dentin blocks with $S$. mutans impregnation and primer application were placed into tubes with $2 \mathrm{~mL}$ of CPW, and the bacteria were harvested by the sonication method. Then, the bacterial suspensions were serially diluted in CPW and plated on $\mathrm{BHI}$ agar plates. After incubating the agar plates at $37^{\circ} \mathrm{C}$ with $5 \% \mathrm{CO}_{2}$ for 3 days, the number of colonies were counted, as in a previous study. ${ }^{36}$ Six dentin blocks were tested for each primer $(n=6)$.

\section{Statistical analysis}

One-way and two-way analyses of variance were performed to detect the significant effects of the variables. The Kolmogoroff-Smirnoff test was used to examine the normal distribution of data. Tukey's multiple comparison was used to compare the data at $P$ value of 0.05 . Standard deviations (SD) served as the estimate for measurement uncertainties for each method.

\section{RESULTS}

Dentin shear bond strengths using the six primers are shown in Figure 1 (mean $\pm \mathrm{SD} ; n=10$ ). The same SBMP adhesive was used for all groups, and the only difference was the primer. All six groups were assessed via the Kolmogoroff-Smirnoff test and all groups presented normal distribution in the data. All six groups were not significantly different from each other $(P=0.389)$. The results showed that adding DMADDM and QADM into the primer did not adversely affect the dentin bond strength $(P>0.1)$.

The antibacterial effects of the six primers were measured via ADT and the results are plotted in Figure 2 (mean \pm SD; $n=6$ ). Control primer had minimal inhibition zones. The antibacterial primers containing QADM or DMADDM had significantly larger inhibition zones $(P<0.05)$. Primers containing $7.5 \%$ or $10 \%$ DMADDM had inhibition zone sizes nearly an order of magnitude greater than that of control primer $(P<0.05)$.

Representative SEM micrographs of dentin blocks and S. mutans impregnation are shown in Figure 3: (a) examples of dentinal tubules "T" before S. mutans impregnation, (b) dentin with S. mutans impregnated into dentinal tubules, and (c) higher magnification showing $S$. mutans inside dentinal tubules. These images demonstrated that $S$. mutans were impregnated into the tubules.

Typical live/dead staining images of $S$. mutans-impregnated dentin after applying the six primers are shown in Figure $4 \mathrm{a}-4 \mathrm{f}$. Live bacteria were stained green, and compromised bacteria were stained red. Dentin with SBMP control primer had much more live S. mutans than 


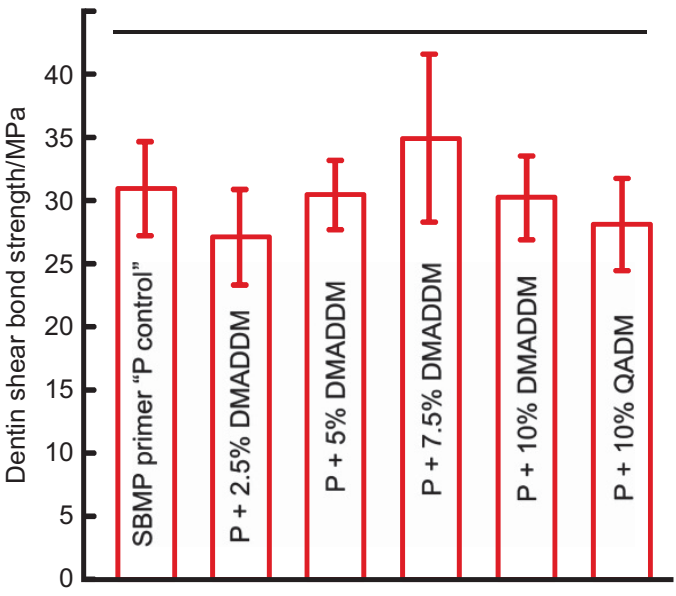

Figure 1 Dentin shear bond strength. Mean $\pm \mathrm{SD} ; n=10$. Horizontal line indicates no significant differences $(P>0.1)$. Therefore, adding DMADDM and QADM into primer did not compromise the dentin bond strength. DMADDM, dimethylaminododecyl methacrylate; QADM, quaternary ammonium dimethacrylate; SD, standard deviations.

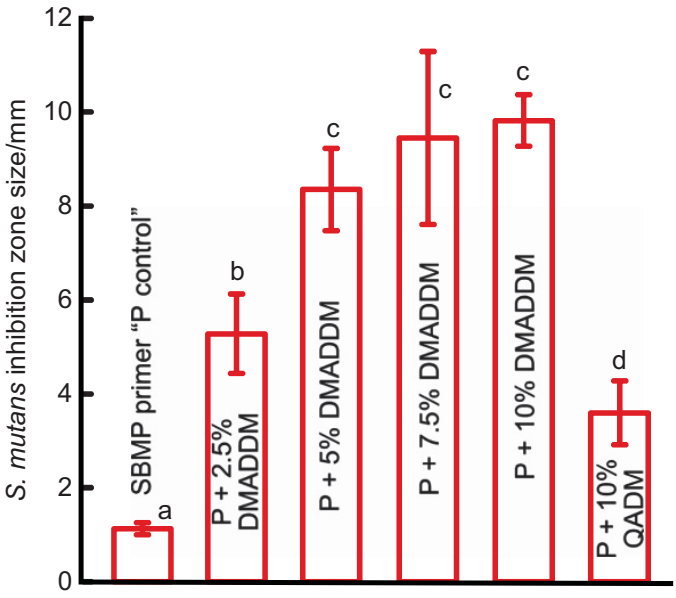

Figure 2 Antibacterial activity of uncured primers in agar disk diffusion test. The control group showed a small inhibition zone. The QADM group had a wider inhibition zone. DMADDM yielded much wider inhibition zones for primers as the DMADDM concentration was increased. Each value is mean $\pm \operatorname{SD}(n=6)$. Values with dissimilar letters $(a-d)$ are significantly different from each other $(P<0.05)$. DMADDM, dimethylaminododecyl methacrylate; QADM, quaternary ammonium dimethacrylate; SD, standard deviations.

the other groups. Primer with QADM resulted in a remarkable increase in red staining of compromised S. mutans. Primers containing DMADDM had the most red staining, indicating that primers containing DMADDM likely killed the bacteria, and that DMADDM had a stronger antibacterial effect than QADM.

The results of methods to harvest bacteria from dentin blocks impregnated with $S$. mutans are plotted in Figure 5 (mean $\pm S D$; $n=6$ ). The sonication method harvested nearly all the $S$. mutans in the dentin blocks. After sonication, the residual bacteria in dentin harvested by cutting the dentin into pieces and homogenization was four orders of magnitude lower than that harvested by sonication method. The total CFU in the dentin blocks were estimated as the CFU harvested by sonication plus the residual bacteria harvested by
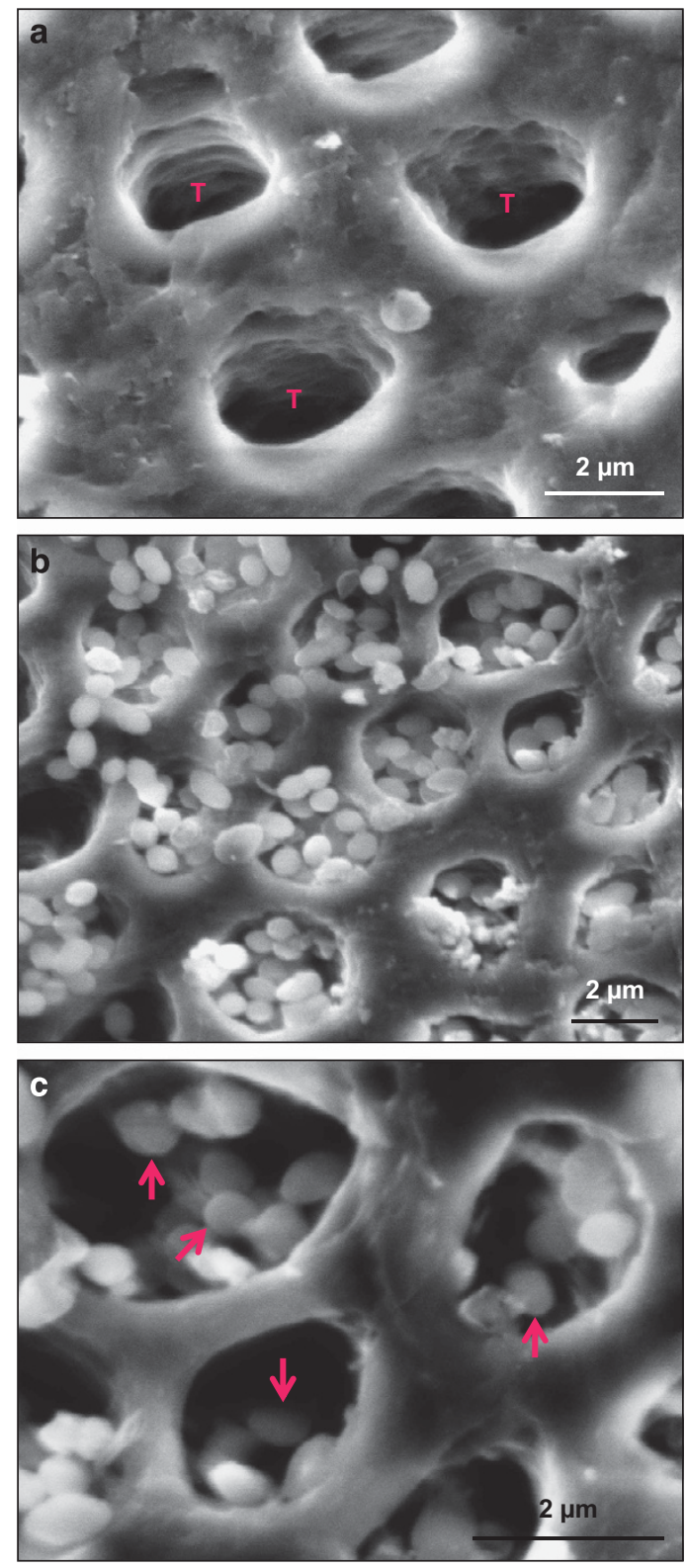

Figure 3 Representative SEM images of dentin and $S$. mutans impregnation. (a) Dentinal tubules before $S$. mutans impregnation, and (b) and (c) S. mutans were impregnated into dentinal tubules, at increasing magnification. T: dentinal tubules. Arrows indicate $S$. mutans in tubules. SEM, scanning electron microscopy.

cutting the dentin into small pieces and homogenization. Hence, the CFU harvested by sonication was equal to $\sim 99.99 \%$ of the total CFU in the dentin blocks. These results demonstrated that sonication was an effective bacterial harvesting method.

Figure 6 plots the CFU of $S$. mutans impregnated in dentin and measured using the sonication method for the six primer groups (mean $\pm \mathrm{SD} ; n=6)$. Dentin with SBMP primer control had the highest CFU. CFU values from dentin treated with primers containing QADM were significantly lower than that for control $(P<0.05)$. Increasing the DMADDM mass fraction in the primer significantly decreased the bacteria CFU $(P<0.05)$. CFU of viable bacteria inside dentin for the primer with $10 \%$ DMADDM was three orders of magnitude lower than that of the SBMP control primer. At the same $10 \%$ mass fraction, 

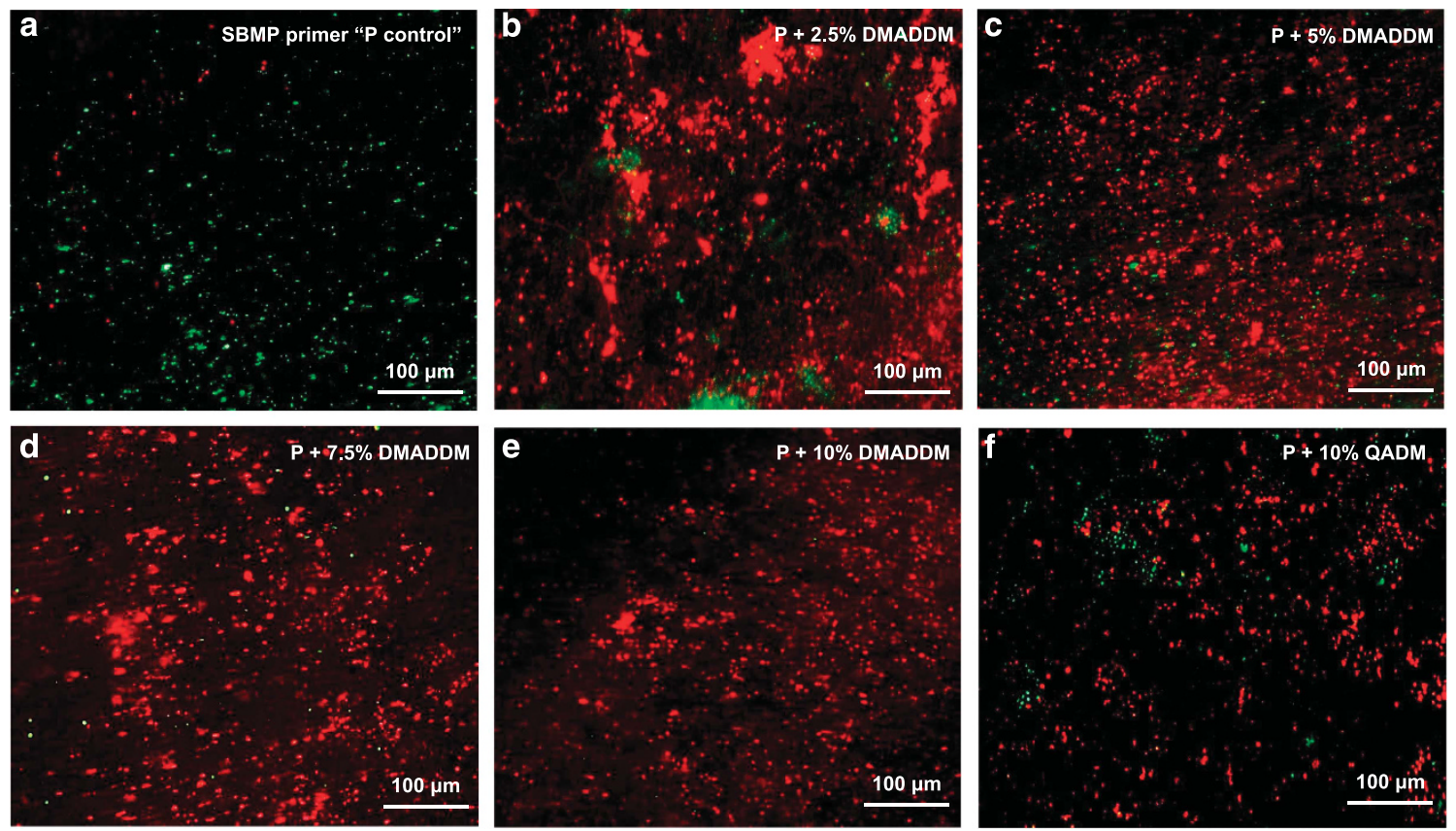

Figure 4 Live/dead $S$. mutans staining images of dentin blocks treated with the six primers. (a) SBMP primer 'P' (control), (b) $P+2.5 \%$ (by mass) DMADDM, (c) P+5\% DMADDM, (d) P+7.5\% DMADDM, (e) P+10\% DMADDM, (f) P+10\% QADM. Live bacteria were stained green, and compromised bacteria were stained red. Dentin with SBMP control primer had primarily live bacteria. In contrast, all the four groups containing DMADDM had mostly red bacteria, indicating that DMADDM-containing primer had a potent antibacterial activity to kill residual bacteria in dentin in the prepared tooth cavity. DMADDM, dimethylaminododecyl methacrylate; QADM, quaternary ammonium dimethacrylate.

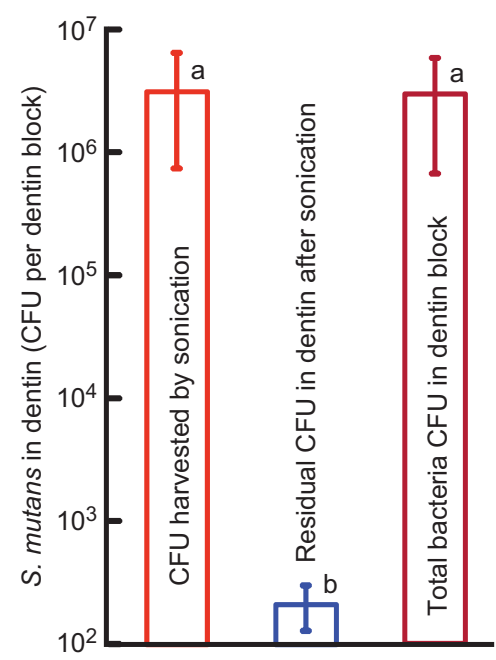

Figure 5 Harvesting $S$. mutans that had been impregnated in dentin. The sonication method harvested nearly all the $S$. mutans in dentin (mean \pm SD; $n=6)$. The total CFU in dentin=the CFU harvested by sonication+the residual CFU in dentin. The residual bacteria in dentin after sonication were harvested by cutting the dentin into pieces and homogenization. CFU harvested by sonication was $99.99 \%$ of the total CFU. Therefore, the simple sonication method could be used to harvest the bacteria in dentin blocks. Values with dissimilar letters $(a, b)$ are significantly different from each other $(P<0.05)$. CFU, colony-forming units; SD, standard deviations.

the CFU of viable bacteria inside dentin for DMADDM group was much lower than that for QADM group, showing that DMADDM had a much higher killing efficacy than QADM.

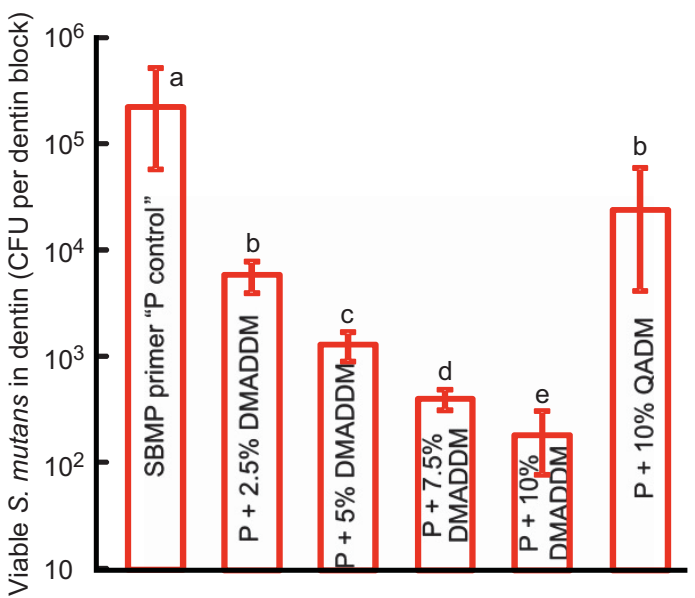

Figure 6 Effects of different primers on S. mutans CFU in dentin blocks harvested by sonication (mean $\pm \mathrm{SD} ; n=6$ ). DMADDM-containing primer effectively killed the bacteria impregnated in dentin blocks, reducing the culturable bacteria CFU harvested from dentin by three orders of magnitude compared with commercial control, and by two orders of magnitude compared with a previous QADM. Values with dissimilar letters (a-e) are significantly different from each other $(P<0.05)$. CFU, colony-forming units; DMADDM, dimethylaminododecyl methacrylate; QADM, quaternary ammonium dimethacrylate; SD, standard deviations.

\section{DISCUSSION}

This study showed that antibacterial primer containing DMADDM was much more effective than QADM in killing bacteria impregnated into dentin blocks. It is known that caries is a dietary carbohydratemodified bacterial infectious disease. ${ }^{43}$ Its key feature is a dietary 
carbohydrate-induced enrichment of the plaque microbiota with organisms that would produce acid. ${ }^{43-44}$ S. mutans have a vital role in the process of primary and secondary caries. ${ }^{43-44}$ Residual bacteria often exist in prepared tooth cavities, and new bacteria can invade the tooth-restoration margins to cause secondary caries, which is a main reason for restoration failure. ${ }^{15,45}$ Since dental primer directly contacts dentinal tubules, adding antibacterial agent into primer could help inhibit the growth of residual and invading bacteria. ${ }^{16}$ Thus, primer could serve as a promising vehicle for delivering antimicrobial agents. ${ }^{27,36}$

DMADDM is a recently synthesized quaternary ammonium monomer with an alkyl chain length of $12 .{ }^{37}$ The antibacterial mechanism of quaternary ammonium salts is that they can cause bacteria lysis by binding to cell membrane. ${ }^{34}$ When the negatively charged bacterial cell contacts the positive charge of quaternary amine $\mathrm{N}^{+}$, the electric balance is disturbed to cause cytoplasmic leakage. ${ }^{46}$ In previous studies, many novel agents (MDPB, DMAE-CB, chlorhexidine particles, silver nanoparticles, and so on) were developed with antibacterial functions. ${ }^{29,35-37,47-50} \mathrm{QADM}$ is a quaternary ammonium dimethacrylate with less negative impact than a monomethacrylate on mechanical properties when it is incorporated and co-polymerized in a resin. In addition, a dimethacrylate is expected to have minimal monomer leach-out due to reactive groups on both ends of the molecule, as compared with a monomethacylate. ${ }^{29,35}$ The present study showed that DMADDM was even more strongly antibacterial than QADM. DMADDM increased the bacteria inhibition zone and killed bacteria in dentinal tubules, which indicated the capability of cavity cleansing and disinfection by killing residual bacterial in tooth cavities.

The reason that DMADDM had a stronger antibacterial effect may be related to its longer carbon chain length. The carbon chain of quaternary ammonium needs to be long enough to penetrate the cell membranes to kill bacteria. ${ }^{51-52}$ DMADDM had a chain length of 12 , while QADM had a chain length of 2. Previous studies also indicated that the antibacterial activity would be enhanced if the carbon chain length was increased. For example, Xie et al. showed that increasing the chain length of quaternary ammonium monomer in glass ionomers significantly increased the antibacterial potency. ${ }^{53}$ Cheng et al. showed that DMADDM with a chain length of 12 possessed a much stronger antibacterial function than another monomer with a chain length of $6 .^{37}$ These results are consistent with the present study on killing bacteria inside dentinal tubules via antibacterial primers, in which DMADDM was much more potent than QADM. Whether the killing of bacteria inside dentinal tubules can be further enhanced if the chain length is increased to $>12$ requires a further study.

Residual bacteria usually exist inside dentinal tubules after tooth cavity preparation, which could lead to pulpal damage. ${ }^{16}$ When the seal of a restoration is maintained, the bacterial numbers will likely diminish considerably, and caries is not likely to progress. However, for deep lesions approximating pulpal tissues and where infected dentin remains, it would be advantageous if bacterial numbers can be further reduced before restoration placement. Two problems exist regarding the use of traditional cavity disinfectants. First, whether traditional cavity disinfectants would reduce the adhesive bond strength remains a concern. ${ }^{54-55}$ Second, traditional cavity disinfectants can not completely eliminate the viable microorganisms in the tooth cavity, and the antibacterial effect is not maintained for a long time. ${ }^{56}$ In this regard, an antibacterial primer containing a quaternary ammonium monomer is advantageous. First, it directly contacts the tooth structure and flows into the dentinal tubules, and could kill residual bacteria in dentin. Second, after photo-polymerization, the quaternary ammonium monomer is co-polymerized and remains in place to provide a long-term antibacterial effect. The present study showed that the novel DMADDM-containing primer could kill the bacteria impregnated in dentin blocks, reducing the viable bacteria CFU harvested from dentin by three orders of magnitude, compared with a commercial primer. This was achieved without negatively affecting the dentin bond strength. Therefore, DMADDM may be promising for use in various primers and bonding agents, as well as in cements, sealants and other dental resins.

It should be noted that the surrounding dental tissues as well as food and saliva may affect the antibacterial function. Carious dentin is often sclerotic with a poor permeability due to occlusion by whitlockite crystals; how the antibacterial primer can enter and penetrate such tubules to kill residual bacteria remains to be investigated. In addition, for deep cavities, whether the antibacterial primer will reach the pulp and what effect it will have on pulpal tissues need to be examined. Furthermore, at the restoration margin where the antibacterial bonding agent could potentially inhibit the invading bacteria, saliva could compromise the antibacterial efficacy. Indeed, coating the antibacterial resin with saliva moderately reduced the antibacterial activity, although biofilm growth and acid production were still substantially reduced. ${ }^{57}$ Further studies are needed to investigate these and other clinically relevant issues regarding the in vivo efficacy and long-term durability of antibacterial bonding agents.

\section{CONCLUSIONS}

The present study investigated the effect of DMADDM mass fraction in primer on the efficacy of killing bacteria inside dentin blocks. The effect of DMADDM was compared with QADM on the killing of S. mutans impregnated into dentin blocks. The hypotheses were proven that while both DMADDM-containing primer and QADM-containing primer killed S. mutan inside dentin, the DMADDM-containing primer was much more potent; that increasing the DMADDM content in primer increased the efficacy of killing bacteria inside dentin; and that the dentin bond strength matched that of control without DMADDM. The novel DMADDM-containing primer reduced the viable bacterial $\mathrm{CFU}$ in dentin by three orders of magnitude, compared with a commercial primer. DMADDM-containing primer reduced bacteria $\mathrm{CFU}$ in dentin by two orders of magnitude, compared with the previous QADM. Therefore, DMADDM is a promising antibacterial monomer for use in primers as well as other resins such as adhesives and cements.

\section{ACKNOWLEDGEMENTS}

This study was supported by NIH R01 DE17974 (Hockin HK Xu), a scholarship from West China School of Stomatology (Chen Chen), Program for New Century Excellent Talents in University (Lei Cheng), Youth Grant of Science and Technology Department of Sichuan Province China (2014JQ0033) (Lei Cheng), a bridge fund from University of Maryland Baltimore School of Dentistry (Hockin $\mathrm{HK} \mathrm{Xu}$ ), and a seed grant from University of Maryland Baltimore (Hockin HK Xu). There is no conflict of interest.

\section{DISCLAIMER}

Certain commercial materials and equipment are identified to specify the experimental procedure. This does not imply recommendation or endorsement by NIST or that the material or equipment identified is necessarily the best available for the purpose. Official contribution of the National Institute of Standards and Technology (NIST); not subject to copyright in the United States. 
1 ten Cate JM. Biofilms, a new approach to the microbiology of dental plaque. Odontology 2006; 94(1): 1-9.

2 Selwitz RH, Ismail AI, Pitts NB. Dental caries. Lancet 2007; 369(9555): 51-59.

3 Beazoglou T, Eklund S, Heffley $D$ et al. Economic impact of regulating the use of amalgam restorations. Public Health Rep 2007; 122(5): 657-663.

4 Bayne SC, Thompson JY, Swift EJ Jr et al. A characterization of first-generation flowable composites. J Am Dent Assoc 1998; 129(5): 567-577.

5 Lim BS, Ferracane JL, Sakaguchi RL et al. Reduction of polymerization contraction stress for dental composites by two-step light-activation. Dent Mater 2002; 18(6): $436-444$.

$6 \mathrm{Xu} \mathrm{X}$, Ling L, Wang $\mathrm{R}$ et al. Formation and characterization of a novel fluoride-releasing dental composite. Dent Mater 2006; 22(11): 1014-1023.

7 Drummond JL. Degradation, fatigue, and failure of resin dental composite materials. J Dent Res 2008; 87(8): 710-719.

8 Imazato S. Bioactive restorative materials with antibacterial effects: new dimension of innovation in restorative dentistry. Dent Mater J 2009; 28(1): 11-19.

9 Amirouche-Korichi A, Mouzali M, Watts DC. Effects of monomer ratios and highly radiopaque fillers on degree of conversion and shrinkage-strain of dental resin composites. Dent Mater 2009; 25(11): 1411-1418.

10 Samuel SP, Li S, Mukherjee I et al. Mechanical properties of experimental dental composites containing a combination of mesoporous and nonporous spherical silica as fillers. Dent Mater 2009; 25(3): 296-301.

11 Ferracane JL. Resin composite - state of the art. Dent Mater 2011; 27(1): 29-38.

12 Mjor IA, Toffenetti F. Secondary caries: a literature review with case reports. Quintessence Int 2000; 31(3): 165-179.

13 Kidd EAM, Banerjee A. What is absence of caries? // Albrektsson TO, Bratthall D, Glantz POJ et al. Tissue preservation in caries treatment. New Malden: Quintessence Publishing, 2001: 69-79.

14 de Almeida Neves A, Coutinho E, Cardoso MV et al. Current concepts and techniques for caries excavation and adhesion to residual dentin. J Adhes Dent 2011; 13(1): 7-22.

15 Sakaguchi RL. Review of the current status and challenges for dental posterior restorative composites: clinical, chemistry, and physical behavior considerations. Summary of discussion from the Portland Composites Symposium (POCOS) June 17-19, 2004, Oregon Health and Science University, Portland, Oregon. Dent Mater 2005; 21(1): 3-6.

16 Imazato S, Kuramoto A, Takahashi $Y$ et al. In vitro antibacterial effects of the dentin primer of Clearfil Protect Bond. Dent Mater 2006; 22(6): 527-532.

17 Murdoch-Kinch CA, McLean ME. Minimally invasive dentistry. J Am Dent Assoc 2003; 134(1): 87-95.

18 Lynch CD, Frazier KB, McConnell RJ et al. Minimally invasive management of dental caries: contemporary teaching of posterior resin-based composite placement in U.S. and Canadian dental schools. J Am Dent Assoc 2011; 142(6): 612-620.

19 Ratledge DK, Kidd EA, Beighton D. A clinical and microbiological study of approximal carious lesions. Part 2: efficacy of caries removal following tunnel and class II cavity preparations. Caries Res 2001; 35(1): 8-11.

20 Spencer $\mathrm{P}$, Wang Y. Adhesive phase separation at the dentin interface under wet bonding conditions. J Biomed Mater Res 2002; 62(3): 447-456.

21 Ritter AV, Swift EJ Jr, Heymann $\mathrm{HO}$ et al. An eight-year clinical evaluation of filled and unfilled one-bottle dental adhesives. J Am Dent Assoc 2009; 140(1): 28-37.

22 Garcia-Godoy F, Kramer N, Feilzer AJ et al. Long-term degradation of enamel and dentin bonds: 6-year results in vitro vs. in vivo. Dent Mater 2010; 26(11): 1113-1118.

23 Pinzon LM, Oguri M, O'Keefe $\mathrm{K}$ et al. Bond strength of adhesives to dentin contaminated with smoker's saliva. Odontology 2010; 98(1): 37-43.

24 Park J, Eslick J, Ye Q et al. The influence of chemical structure on the properties in methacrylate-based dentin adhesives. Dent Mater 2011; 27(11): 1086-1093.

25 Pashley DH, Tay FR, Breschi L et al. State of the art etch-and-rinse adhesives. Dent Mater 2011; 27(1): 1-16.

26 Van Meerbeek B, Yoshihara K, Yoshida $Y$ et al. State of the art of self-etch adhesives. Dent Mater 2011; 27(1): 17-28.

27 Imazato S. Antibacterial properties of resin composites and dentin bonding systems. Dent Mater 2003; 19(6): 449-457.

28 Tezvergil-Mutluay A, Agee KA, Uchiyama T et al. The inhibitory effects of quaternary ammonium methacrylates on soluble and matrix-bound MMPs. J Dent Res 2011; 90(4): 535-540.

29 Antonucci JM, Zeiger DN, Tang $\mathrm{K}$ et al. Synthesis and characterization of dimethacrylates containing quaternary ammonium functionalities for dental applications. Dent Mater 2012; 28(2): 219-228.

$30 \mathrm{Xu} \mathrm{X}$, Wang Y, Liao S et al. Synthesis and characterization of antibacterial dental monomers and composites. J Biomed Mater Res B Appl Biomater 2012; 100(4): 1151-1162.

31 Weng $Y$, Howard L, Guo X et al. A novel antibacterial resin composite for improved dental restoratives. J Mater Sci Mater Med 2012; 23(6): 1553-1561.

32 Imazato S, Kinomoto $\mathrm{Y}$, Tarumi $\mathrm{H}$ et al. Antibacterial activity and bonding characteristics of an adhesive resin containing antibacterial monomer MDPB. Dent Mater 2003; 19(4): 313-319.
$33 \mathrm{Li} \mathrm{F}$, Chen J, Chai Z et al. Effects of a dental adhesive incorporating antibacterial monomer on the growth, adherence and membrane integrity of Streptococcus mutans. J Dent 2009; 37(4): 289-296.

34 Beyth N, Yudovin-Farber I, Bahir R et al. Antibacterial activity of dental composites containing quaternary ammonium polyethylenimine nanoparticles against Streptococcus mutans. Biomaterials 2006; 27(21): 3995-4002.

35 Cheng L, Weir MD, Xu HH et al. Antibacterial amorphous calcium phosphate nanocomposites with a quaternary ammonium dimethacrylate and silver nanoparticles. Dent Mater 2012; 28(5): 561-572.

36 Cheng L, Zhang K, Weir MD et al. Effects of antibacterial primers with quaternary ammonium and nano-silver on Streptococcus mutans impregnated in human dentin blocks. Dent Mater 2013; 29(4): 462-472.

37 Cheng L, Weir MD, Zhang $\mathrm{K}$ et al. Dental primer and adhesive containing a new antibacterial quaternary ammonium monomer dimethylaminododecyl methacrylate. J Dent 2013; 41(4): 345-355.

$38 \mathrm{Li} \mathrm{F}$, Weir MD, Chen J et al. Effect of charge density of bonding agent containing a new quaternary ammonium methacrylate on antibacterial and bonding properties. Dent Mater 2014; 30(4): 433-441.

39 Zhang K, Cheng L, Wu EJ et al. Effect of water-ageing on dentine bond strength and anti-biofilm activity of bonding agent containing new monomer dimethylaminododecyl methacrylate. J Dent 2013; 41(6): 504-513.

40 Antonucci JM, O'Donnell JN, Schumacher GE et al. Amorphous calcium phosphate composites and their effect on composite-adhesive-dentin bonding. J Adhes Sci Technol 2009; 23(7): 1133-1147.

41 Melo MA, Cheng L, Zhang $\mathrm{K}$ et al. Novel dental adhesives containing nanoparticles of silver and amorphous calcium phosphate. Dent Mater 2013; 29(2): 199-210.

42 Zhang K, Melo MA, Cheng L et al. Effect of quaternary ammonium and silver nanoparticle-containing adhesives on dentin bond strength and dental plaque microcosm biofilms. Dent Mater 2012; 28(8): 842-852.

43 van Houte J. Role of micro-organisms in caries etiology. J Dent Res 1994; 73(3): 672-681.

44 Takahashi N, Nyvad B. The role of bacteria in the caries process: ecological perspectives. J Dent Res 2011; 90(3): 294-303.

45 Jokstad A, Bayne S, Blunck U et al. Quality of dental restorations. FDI Commission Project 2-95. Int Dent J 2001; 51(3): 117-158.

46 Namba N, Yoshida Y, Nagaoka N et al. Antibacterial effect of bactericide immobilized in resin matrix. Dent Mater 2009; 25 (4): 424-430.

47 Imazato S, Kinomoto $\mathrm{Y}$, Tarumi $\mathrm{H}$ et al. Incorporation of antibacterial monomer MDPB into dentin primer. J Dent Res 1997; 76(3): 768-772.

48 Imazato S, Ehara A, Torii M et al. Antibacterial activity of dentine primer containing MDPB after curing. J Dent 1998; 26(3): 267-271.

49 Hiraishi N, Yiu CK, King NM et al. Effect of chlorhexidine incorporation into a selfetching primer on dentine bond strength of a luting cement. J Dent 2010; 38(6): 496-502.

50 Brambilla E, Ionescu A, Fadini L et al. Influence of MDPB-containing primer on Streptococcus mutans biofilm formation in simulated class I restorations. J Adhes Dent 2013; 15(5): 431-438.

51 Tiller JC, Liao CJ, Lewis $\mathrm{K}$ et al. Designing surfaces that kill bacteria on contact. Proc Natl Acad Sci USA 2001; 98(11): 5981-5985.

52 Murata H, Koepsel RR, Matyjaszewski K et al. Permanent, non-leaching antibacterial surface-2: how high density cationic surfaces kill bacterial cells. Biomaterials 2007; 28(32): 4870-4879.

53 Xie D, Weng Y, Guo X et al. Preparation and evaluation of a novel glass-ionomer cement with antibacterial functions. Dent Mater 2011; 27(5): 487-496.

54 Vieira Rde S, da Silva IA Jr. Bond strength to primary tooth dentin following disinfection with a chlorhexidine solution: an in vitro study. Pediatr Dent 2003; 25(1): 49-52.

55 Ercan E, Erdemir A, Zorba YO et al. Effect of different cavity disinfectants on shear bond strength of composite resin to dentin. J Adhes Dent 2009; 11(5): 343-346.

56 Borges FM, de Melo MA, Lima JP et al. Antimicrobial effect of chlorhexidine digluconate in dentin: In vitro and in situ study. J Conserv Dent 2012; 15(1): 22-26.

$57 \mathrm{Li} \mathrm{F}$, Weir MD, Fouad AF et al. Effect of salivary pellicle on antibacterial activity of nove antibacterial dental adhesives using a dental plaque microcosm biofilm model. Dent Mater 2014; 30(2): 182-191.

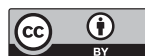

This work is licensed under a Creative Commons Attribution 4.0 International License. The images or other third party material in this article are included in the article's Creative Commons license, unless indicated otherwise in the credit line; if the material is not included under the Creative Commons license, users will need to obtain permission from the license holder to reproduce the material. To view a copy of this license, visit http://creativecommons.org/licenses/by/4.0/

(C) The Author(s) 2016 